\title{
Congruencia programática en el Cono Sur. Argentina, Chile y Uruguay en perspectiva comparada
}

\author{
Mario Herrera ${ }^{1}$ \\ Mauricio Morales ${ }^{2}$
}

\begin{abstract}
Los sistemas de partidos institucionalizados pueden convivir con una alta incongruencia programática entre élite y ciudadanía. Por el contrario, sistemas de partidos menos institucionalizados podrían estar en la posición de generar una mayor congruencia programática. Analizamos esta aparente anomalía en el Cono Sur de América Latina, comparando Argentina, Chile y Uruguay. Metodológicamente, utilizamos tres medidas de congruencia: promedios, comparación de distribuciones (many to many), distancia entre los votantes y un representante típico (many to one). Sobre esta última medida diseñamos modelos de regresión múltiple para identificar las variables que predicen mayor congruencia. Concluimos que en sistemas estables las preferencias electorales se mantienen a pesar de la distancia entre élite y votantes en algunos ejes de competencia, siendo Uruguay el caso más representativo.

Palabras claves: congruencia programática; sistemas de partidos; institucionalización; cono sur; elite
\end{abstract}

\section{Introducción ${ }^{3}$}

Desde la teoría de gobierno responsable, se asume que una buena democracia se construye sobre la base de ciudadanos que eligen a sus representantes según sus posturas programáticas y que, una vez en el gobierno, llevan a cabo políticas públicas que responden a ese programa (Adams, 2001). Muchas veces se asocia un gobierno de partido responsable con los niveles de institucionalización de los sistemas de partidos de acuerdo a la clásica definición de Mainwaring y Scully (1995). Es decir, sistemas de partidos con bajos niveles de volatilidad electoral y con alta raigambre social. Los trabajos de Kitschelt (2000) y de Luna y Zechmeister (2005) iluminan esta combinación ideal para Europa del Este y América Latina respectivamente.

Tal combinación ideal entre sistemas de partidos institucionalizados y altos grados de congruencia entre élite y votantes conduce a una "buena representación". Los legisladores

\footnotetext{
1 Universidad de Talca - Escuela de Ciencia Política y Administración Pública. Talca, Chile. E-mail: <mherreram@utalca.cl>. Orcid: <https://orcid.org/0000-0001-5453-5594>.

2 Universidad de Talca - Escuela de Ciencia Política y Administración Pública. Talca, Chile. Centro de Estudios de Conflicto y Cohesión Social, Santiago de Chile. Chile. E-mail: <mmoralesq@utalca.cl>. Orcid: <https://orcid.org/0000-0001-6010-9604>.

${ }^{3}$ Este artículo recibió financiamiento del Proyecto Fondecyt N 1180009.
} 
representan las opiniones ciudadanas, y los ciudadanos los votan premiando el compromiso programático (Adams, 2001; Luna, 2007; Kitschelt et al., 2010). En el lado opuesto están los sistemas de partidos en que los representantes, una vez en el poder, apoyan políticas públicas lejanas a las preferencias de sus propios votantes. Es lo que Stokes (2001) define como policy switch, situación que puede darse en sistemas estables o inestables (Weyland, 2004; Morgan, 2007). En este escenario, los votantes abandonan a sus partidos, decidiendo abstenerse en las siguientes elecciones, o respaldando a partidos emergentes. Esto puede concluir con una espiral de desafección, la aparición de zonas marrones que alimentan el déficit institucional (O’Donnell, 1994) o, incluso, con el colapso del sistema de partidos (Morgan, 2007).

Esta literatura, por tanto, ve en la congruencia programática entre partidos y electores una variable central para explicar la estabilidad y -de cierta forma- los niveles de institucionalización de un sistema de partidos. Difícilmente un sistema de partidos se institucionalizará junto con bajos niveles de congruencia programática $y$, al mismo tiempo, un sistema con tales características -casi por definición- es aquel en que se ejerce una "buena representación". Dado que la institucionalización se define principalmente de acuerdo a los niveles de volatilidad electoral e identificación partidaria, ese sistema de partidos se reproducirá sobre la base del compromiso programático permanente entre representantes y votantes.

Tal visión optimista es discutida por la evidencia. En este artículo mostramos que la congruencia programática no se cultiva necesariamente en contextos de alta institucionalización partidaria. Nájera-España y Martínez (2010) muestran esta aparente anomalía para Centroamérica. En el Cono Sur -como argumentamos aquí- sucede algo similar: existen mayores volúmenes de congruencia en Argentina en comparación con Chile y Uruguay. En las mediciones de Mainwaring y Scully (1995) y de Payne et al. (2003), Chile y Uruguay aparecen sistemáticamente superiores a Argentina cuando se considera el índice de institucionalización. De igual manera sucede con el índice de calidad de la democracia elaborado por Levine y Molina (2011). De acuerdo a esta aparente contradicción -sistemas institucionalizados con baja congruencia programática, y sistemas menos institucionalizados con alta congruencia programática- surgen las siguientes preguntas: ¿cómo varían los niveles de congruencia programática en el Cono Sur y, específicamente, en Argentina, Chile y Uruguay?, ¿qué tanta congruencia programática existe entre las élites y los votantes de estos países al examinar el eje ideológico izquierda-derecha?, ¿qué factores políticos y sociales explican las variaciones de la congruencia programática en el Cono Sur?

Parte de esta discusión ha sido abordada por Luna (2007) y Kitschelt et al. (1999). Para ambos, los niveles de institucionalización de los sistemas de partidos no necesariamente van asociados a altos niveles de congruencia programática. En realidad, existen distintos tipos de institucionalización. Por un lado, la institucionalización programática donde se espera la combinación ideal que definimos más arriba. Por otro lado, la institucionalización no programática derivada de vínculos clientelares entre partidos y electores, o producto de 
la mayor incidencia de los liderazgos carismáticos a la hora de definir las adhesiones partidarias. Pero ¿qué sucede en aquellos sistemas de partidos donde las élites reaccionan de una forma ideológicamente más intensa frente a los ejes de competencia, marcando distancia incluso con sus propios representados? El sistema de partidos no verá necesariamente afectados sus niveles de institucionalización, pero sí sus niveles de congruencia programática.

Esta tensión teórica entre institucionalización y congruencia lleva a formular una propuesta alternativa. Probablemente, la congruencia programática no sea exclusiva de los sistemas de partidos estables e institucionalizados. Puede darse en ambientes de menor institucionalización, fortaleciendo la idea de que congruencia e institucionalización no tienen -necesariamente- una relación lineal. Entonces, lo que debe examinarse con mayor detalle son las características de la congruencia programática que predomina en cada país. En este artículo avanzamos hacia ese objetivo, mostrando no sólo el volumen de congruencia medido a través de estadística descriptiva, sino que también proponemos un análisis sobre la composición de la congruencia en cada país recurriendo a modelos de regresión multivariados.

Utilizamos datos de encuestas a representantes de Argentina, Chile y Uruguay, y de encuestas a ciudadanos aplicadas casi de manera simultánea en 2013. El proyecto fue financiado por el International Development Research Centre (IDRC). La disposición de encuestas a élites y a ciudadanos nos permite -además- avanzar en una segunda dimensión. Generalmente los estudios de congruencia se concentran más en el volumen que en la composición de la misma. Lo que hacemos en este artículo es identificar los factores políticos y sociales que explican mayores o menores niveles de congruencia. Para eso, comparamos a un representante "promedio" con la opinión de los ciudadanos.

El texto se divide en cuatro secciones. En la primera mostramos la teoría sobre congruencia programática y, específicamente, su relación con la institucionalización partidaria. En la segunda detallamos la metodología a utilizar. La tercera sección corresponde a la descripción del método y de los datos, colocando especial atención a las formas de medir congruencia programática. Lo hacemos mediante comparación de promedios, comparación de distribuciones (many to many) y comparación de un legislador promedio versus los ciudadanos (many to one). En esto seguimos las sugerencias de Golder y Stramski (2010). En la cuarta sección sintetizamos nuestras principales conclusiones.

\section{Teoría}

Si entendemos por representación política aquel proceso en que los representados votan por candidatos que reflejan sus posiciones ideológicas, y que esos candidatos -una vez en el cargo- legislen respetando dichas posiciones (Pitkin, 1967), la representación -o la "buena" representación- supone un sistema de partidos ordenado en algún eje de competencia. Siguiendo a Powell (2004), y específicamente el enfoque de la congruencia 
programática para entender la representación política, los legisladores o representantes debieran ser responsables al momento de generar políticas públicas. Es decir, acoplar las preferencias temáticas de los ciudadanos con la producción legislativa. Dicho en otras palabras, el enfoque de la congruencia programática va más allá de la simple representación descriptiva que prioriza la forma en que los votos inciden en la composición de las legislaturas. En su lugar, subraya la representación sustantiva, que equivale a la traducción de los intereses ciudadanos en políticas públicas.

¿Cómo se procesa la representación política? Para responder esta pregunta existen, al menos, dos modelos. El primero corresponde a la representación por mandato. Acá los representantes generan políticas públicas en función de los intereses de sus representados. El segundo corresponde a la representación por cesión o delegación. En este modelo los representantes producen políticas públicas sin tener en cuenta -necesariamente- las preferencias de sus electores. Más bien, generan esas políticas pensando en el beneficio para sus votantes, sin importar si esas políticas se acoplan o no a los intereses de sus electores. En ambos modelos, siguiendo a Przeworski, Ortega y Gordon (1997), los representantes están expuestos al accountability vertical de los ciudadanos que los confirmarán o sacarán del poder en función de sus promesas de campaña. Idealmente, la combinación entre responsabilidad de los políticos y accountability ciudadano conduce a mejores democracias. Naturalmente, el vicio de la representación por mandato, de acuerdo con O'Donnel (1994), es el tránsito hacia democracias delegativas.

Siguiendo a Adams (2001), un "gobierno de partido responsable" es aquel donde no sólo existe congruencia programática entre representantes y representados, sino que también una divergencia entre las propias élites partidarias. En términos más sencillos, el modelo de partido responsable supone homogeneidad programática entre partidos y votantes, y heterogeneidad programática entre partidos. Esto se traduce en preferencias electorales cristalizadas y estables en el tiempo. De acuerdo a Kitschelt (2000) y Kitschelt et al. (2010), tal estabilidad contribuye a la consolidación de la democracia. A preferencias estables y a partidos responsables, mayor accountability intertemporal y mayor calidad de la democracia. Teóricamente, entonces, una alta congruencia programática entre partidos y votantes reduce el espacio para candidaturas externas a los partidos tradicionales, fortaleciendo lo que Mainwaring y Scully (1995) denominan institucionalización de los sistemas de partidos.

Esta ecuación optimista ha sido respaldada por la evidencia empírica. Así, al menos, se desprende del trabajo de Luna y Zechmeister (2005 y 2010) que comparan los sistemas de partidos latinoamericanos. Concluyen que, efectivamente, las democracias donde se reproduce la congruencia programática son las más estables y con sistemas de partidos institucionalizados. Aunque los representantes suelen ser más polarizados que sus propios votantes en el eje izquierda-derecha (Kitschelt et al., 1999) -cosa que no necesariamente sucede en los sistemas de partidos europeos (Dalton, 1985; Thomassen y Schmitt, 1999; Belchior, 2010)- esas distancias ideológicas entre representantes y representados no se 
traducen en una brecha de representación. Simplemente, los legisladores son más intensos en sus preferencias ideológicas a fin de marcar la identidad de su etiqueta partidaria, distinguiéndose de otros legisladores (Morales, 2014).

Por tanto, al existir partidos heterogéneos en un determinado eje de competencia y con votantes cautivos, lo esperable es que esos partidos sobrevivan junto con la estabilidad del sistema (Powell, 2001). A esto se añade -como condición adicional- cierto grado de cohesión partidaria. Es decir, que los legisladores del partido sean relativamente homogéneos en cuanto a sus posturas con el fin de demarcar claramente su espacio de competencia (Hawkins y Morgenstern, 2000, p. 145).

De acuerdo con la ecuación descrita, el ciclo teórico arrancaría con representantes capaces de representar sustantivamente a sus votantes. Es decir, ejecutar políticas públicas en función de las preferencias de los electores. A cambio, los votantes premiarían a esos partidos, contribuyendo así a la institucionalización del sistema de partidos por efecto de la alta identificación partidaria y la baja volatilidad electoral. En este contexto, y siguiendo a Adams (2001), podría hablarse de un gobierno de partido responsable, lo que finalmente, de acuerdo con el índice elaborado por Levine y Molina (2007), impactaría positivamente en la calidad de la democracia. El ciclo cierra con un sistema de accountability vertical y horizontal. Es decir, con votantes que evalúan a sus representantes en las elecciones, y con instituciones encargadas de fiscalizar los actos de gobierno.

Sin embargo, y como discutimos en este artículo, este ciclo optimista no se reproduce necesariamente de la forma esperada. Un sistema de partidos institucionalizado puede convivir con bajos niveles de congruencia programática, mientras que un sistema menos institucionalizado puede presentar altos niveles de congruencia. Por tanto, la congruencia programática se puede cultivar en ambientes democráticos estables, y en contextos no esperados por el modelo teórico original. Estudios recientes han demostrado que es normal que las posiciones de políticas públicas de la élite difieran de las que tiene la ciudadanía (Warwick, 2016).

¿Por qué se asume que la congruencia programática podría funcionar como predictor de la estabilidad política y, específicamente, como estimulante de la institucionalización partidaria? Partamos diciendo que, según Kitschelt et al. (2010), existen tres tipos de vínculo entre partido y elector. En primer lugar, los vínculos clientelares suponen un intercambio de favores por votos en una relación patrón-cliente. En segundo lugar, el vínculo carismático se basa en los atributos personales del candidato que son valorados por sus votantes. En tercer lugar, está el vínculo programático, que supone la oferta de paquetes de políticas de los partidos a los ciudadanos, y éstos los premian con su voto. De acuerdo con Kitschelt et al. (2010) habrá congruencia programática si las políticas que prefieren los votantes son similares a las que defienden los partidos. La congruencia, entonces, observa la distancia entre la oferta programática del congresista y la preferencia del votante.

Ahora, ¿cuáles son los determinantes de la congruencia programática? EspañaNájera y Martínez (2010) argumentan que los países con menores niveles de PIB per cápita 
debieran tener mayores vínculos clientelares, mientras que en los países más ricos se debieran cultivar los vínculos programáticos, pues existe mayor acceso a la educación y, por tanto, predominan votantes más informados (España-Nájera y Martínez, 2010).

Otro predictor son las tasas de reelección de legisladores. La hipótesis es que la experiencia legislativa impacta positivamente en la congruencia programática. Legisladores con mayor experiencia tienden a conocer de mejor forma las preferencias de sus votantes, acomodándose a sus percepciones (España-Nájera y Martínez, 2010).

Ames y Smith (2010), estudiando el caso de Brasil, identifican como predictores la aprobación al presidente y el apoyo a la democracia. Concluyen que en escenarios de alta aprobación y apoyo al régimen la congruencia programática se reproduce a mayor velocidad. En cambio, un contexto de baja aprobación presidencial supone un distanciamiento entre las opiniones ciudadanas y de los partidos políticos.

La literatura identifica -además- un conjunto de variables institucionales, como los sistemas electorales. Para algunos, los sistemas proporcionales estimulan la formación de partidos y, por ende, la oferta programática (Giger et al., 2011; Dalton, 1985; Huber y Powell, 1994; Wessels, 1999). Esto haría que los partidos representen todo el arco ideológico que acompaña a los votantes debido a la pluralidad de la oferta política. Para otros, en cambio, son los sistemas de mayoría los que generan mayor congruencia. Esto, porque necesariamente deben cautivar al votante mediano para ganar las elecciones (Blais y Bodet, 2006; Powell, 2009; Golder y Lloyd, 2014). Los sistemas proporcionales, en cambio, generan incentivos para la emergencia de más partidos en una competencia multidimensional. Por tanto, se abre espacio para la aparición de partidos extremos que, dada esa condición, se alejan del votante mediano y -como consecuencia- generan una baja congruencia programática.

De esta forma, la congruencia programática ha sido estudiada desde las condiciones estructurales e institucionales de los países, las características socioeconómicas y sociodemográficas de los electores, y variables coyunturales asociadas al ciclo económico y a los niveles de aprobación presidencial. ¿Y qué se ha dicho sobre la congruencia programática en el Cono Sur? Existe cierto consenso respecto a que Chile posee niveles de congruencia programática más altos que el resto de los países de la región (Luna y Zechmeister, 2010; Siavelis, 2009). No obstante, literatura más reciente cuestiona esa afirmación. Sobre la base de una comparación de ciudadanos y legisladores según cada coalición política, el resultado es menos alentador. Sistemáticamente se advierte una mayor polarización de la élite que convive con una ciudadanía más moderada (Morales 2014). En el caso de Uruguay, Buquet y Selios (2017) encuentran altos niveles de congruencia programática en valores asociados con la democracia y en la forma de entender a los partidos políticos como agencias centrales para un régimen democrático. Para Argentina, Lupu y Werner (2017) muestran altos niveles de congruencia programática. No obstante, los autores señalan que existen diferencias entre temáticas. Legisladores y ciudadanos tienen mayores niveles de congruencia en temas sociales y de descentralización, mientras que se advierte 
mayor distancia en libertades civiles y percepción de los principales problemas del país. Lupu y Werner (2017) encuentran, además, que el nivel socioeconómico influye en la congruencia programática. Por ejemplo, en política económica, populismo y libertades civiles, existe una mayor congruencia entre los representantes y los votantes de niveles socioeconómicos más altos.

Esta evidencia preliminar lleva a repensar la validez de la ecuación optimista que mostramos más arriba. Al parecer, la relación entre institucionalización y congruencia programática no es necesariamente lineal. Por otro lado, la literatura ha explorado los factores que explican la congruencia programática entre partidos y votantes en función de las características sociales de los electores. Generalmente, los estudios de congruencia se centran en la comparación de preferencias entre élite y ciudadanos sin auscultar en los predictores que anticipan altos o bajos niveles de congruencia. Además, estos estudios suelen privilegiar datos agregados por país, sin profundizar en un análisis por partido y/o coalición. La idea, entonces, es comparar a las élites partidarias con sus propios votantes y con el resto de la población.

\section{Metodología}

¿Cómo medir la congruencia programática? Los estudios sobre representación han incorporado diferentes formas para medirla. Achen (1977) describe las técnicas estadísticas utilizadas por Powell (1974) y Miller y Stokes (1963), quienes correlacionaron las preferencias de representantes y representados. En un trabajo posterior, Achen (1978) propone tres medidas de congruencia: proximity, centralism y responsiveness. Tres décadas después, Golder y Stramski (2010) establecen otras tres medidas. La medida one-to-one compara la distancia en la preferencia de un legislador y de un representado promedio. El principal problema de esta medida es que omite la dispersión de las distribuciones. La medida many-to-one compara la distancia entre una distribución de la ciudadanía con respecto a un legislador promedio. La medida many-to-many, en tanto, compara las distribuciones de representantes y representados. En específico, se enfoca en la distribución de frecuencias acumuladas, observando el área en que ambas distribuciones se sobreponen.

Para este trabajo utilizamos las tres medidas sugeridas por Golder y Stramski (2010), y ponemos atención particularmente en la estrategia many-to-one. En este estudio diseñamos algunos modelos estadísticos de regresión lineal múltiple para identificar las variables socioeconómicas y sociodemográficas que en mayor medida inciden sobre la congruencia programática. Comparando un legislador promedio de cada partido o coalición en los países seleccionados, nuestro objetivo es conocer los determinantes de la congruencia, evaluando si en el Cono Sur existe algún patrón común para los tres casos.

Para medir congruencia contamos con una encuesta aplicada a ciudadanos y élite en Argentina, Chile y Uruguay. El muestreo fue probabilístico en sus tres etapas -región, comuna y vivienda- y con un tamaño muestral de 1200 casos. Las encuestas se realizaron 
entre marzo y abril de 2013. La encuesta a élites -donde se aplicaron preguntas idénticas a la encuesta de ciudadanos- se realizó entre noviembre de 2013 y octubre de 2014. En específico, la muestra incorporó 102 casos en Argentina, 164 en Chile y 121 en Uruguay.

Naturalmente, la variable dependiente es la congruencia programática. Ésta será entendida como la distancia en las preferencias entre representantes y ciudadanos. Se medirá en dos dimensiones recomendadas por la literatura: ideología y satisfacción con la democracia. Para medir ideología utilizamos el autoposicionamiento en el eje izquierdaderecha, con la siguiente pregunta: "Utilizando una escala de 0 a 10, donde 0 representa más de izquierda y 10 más de derecha, ¿en qué posición se ubicaría usted? Para el caso argentino se complementa el análisis con la misma escala aplicada en el eje peronista y antiperonista, bajo la expectativa de que este eje pueda dar resultados más certeros de lo que sucede en la relación partidos y votantes. Para satisfacción con la democracia, elegimos la siguiente pregunta: "¿Cuán satisfecho está usted con el funcionamiento de la democracia en (Argentina, Chile o Uruguay)?". La pregunta tiene cuatro opciones posibles: "Muy satisfecho", "Bastante satisfecho", "Poco satisfecho", "Nada satisfecho".

Para los modelos de regresión multivariada en función de la estrategia many-to-one, se utilizaron como variables independientes -siguiendo la teoría referida anteriormente- el nivel socioeconómico de los encuestados, la identificación partidaria, percepciones de la economía, y el interés en la política. Como variables de control se incluyó el sexo, edad y zona geográfica. El objetivo es conocer cuáles grupos son más congruentes programáticamente.

¿Por qué seleccionamos Chile, Uruguay y Argentina? En primer lugar, porque los datos provienen de encuestas casi simultáneas en los tres países tanto a ciudadanos como a representantes. En segundo lugar, porque los tres países comparten la misma zona geográfica, sin perjuicio de que sean sistemas de partidos distintos. Mientras Uruguay y Chile tienden a ser considerados como sistemas estables e institucionalizados, Argentina está más atrás (Mainwaring y Scully, 1995; Payne et al., 2003). En tercer lugar, los tres países presentan sistemas electorales proporcionales para elegir a los congresistas, pero con distintas magnitudes de distrito y diferentes tipos de listas. Mientras en Uruguay y Argentina se usan listas cerradas y bloqueadas, en Chile se utilizan listas abiertas. En términos jerárquicos, el sistema electoral uruguayo es el más proporcional, seguido del argentino y, finalmente, del chileno. La Tabla 1 señala los sistemas electorales en los tres países. Preliminarmente, se advierte una relación inversa entre congruencia y proporcionalidad en especial por el caso de Uruguay. 
Tabla 1

Sistemas electorales en Argentina, Chile y Uruguay

\begin{tabular}{|l|c|c|c|c|}
\hline País & Sistema electoral & Lista & $\begin{array}{c}\text { Magnitud de } \\
\text { distrito promedio }\end{array}$ & $\begin{array}{c}\text { Institucionalización del } \\
\text { sistema de partidos }\end{array}$ \\
\hline Argentina & Proporcional D'Hondt & $\begin{array}{c}\text { Cerrada y } \\
\text { bloqueada }\end{array}$ & 5,4 & Baja \\
\hline Chile & Proporcional D'Hondt & Abierta & 2 & Alta \\
\hline Uruguay & Proporcional D'Hondt & $\begin{array}{c}\text { Cerrada y } \\
\text { bloqueada }\end{array}$ & 6,1 & Alta \\
\hline
\end{tabular}

Fuente: Elaboración propia con datos de Payne et al. (2003).

\section{Método y datos}

La primera parte del análisis consiste en un estudio descriptivo de la información, comparando promedios y distribuciones. La segunda parte muestra un análisis inferencial basado en la estrategia many-to-one para la dimensión "ideología".

Como adelantamos, la medición one-to-one compara la media de las preferencias de representantes con sus representados. La Tabla 2 recoge esta perspectiva de análisis y muestra los resultados para los tres países seleccionados. Se advierten variaciones entre los casos. Por ejemplo, en Argentina y Chile la diferencia entre la élite y los ciudadanos en el eje izquierda-derecha es sustancialmente inferior en comparación con Uruguay. Acá mientras la élite promedia 3.3, la ciudadanía promedia 6.6. Por tanto, y de acuerdo con este eje, Uruguay sería el país más incongruente del Cono Sur, pero -a su vez- con el sistema de partidos más institucionalizado de la región. Si bien esta medida tiene como limitante la simple comparación de medias agregadas por país, de cualquier forma, da las primeras señales respecto a las diferencias o similitudes entre los casos.

Tabla 2

\section{Congruencia programática en Argentina, Chile y Uruguay. Medida one-to-one}

\begin{tabular}{|c|c|c|c|}
\hline País & Dimensión & Élite & Ciudadanos \\
\hline \multirow{3}{*}{ Argentina } & Izquierda-Derecha & $\begin{array}{c}4,1 \\
(1,8)\end{array}$ & $\begin{array}{c}5,3 \\
(2,2)\end{array}$ \\
\hline & Peronista-Antiperonista & $\begin{array}{c}3,9 \\
(2,7)\end{array}$ & $\begin{array}{c}4,8 \\
(2,9)\end{array}$ \\
\hline & Satisfacción con la Democracia & $\begin{array}{c}2 \\
(0,76)\end{array}$ & $\begin{array}{c}2,3 \\
(0,9)\end{array}$ \\
\hline \multirow{2}{*}{ Chile } & Izquierda-Derecha & $\begin{array}{c}4,6 \\
(2,2)\end{array}$ & $\begin{array}{c}5,1 \\
(2,1)\end{array}$ \\
\hline & Satisfacción con la Democracia & $\begin{array}{c}2,1 \\
(0,7)\end{array}$ & $\begin{array}{c}2,5 \\
(0,7)\end{array}$ \\
\hline \multirow{2}{*}{ Uruguay } & Izquierda-Derecha & $\begin{array}{c}3,3 \\
(1,6)\end{array}$ & $\begin{array}{c}6,6 \\
(2,7)\end{array}$ \\
\hline & Satisfacción con la Democracia & $\begin{array}{c}3,5 \\
(0,6)\end{array}$ & $\begin{array}{c}1,9 \\
(0,8)\end{array}$ \\
\hline
\end{tabular}

Fuente: Elaboración propia con datos de IDRC (2013). Desviación estándar entre paréntesis. 
En la Figura 1 mostramos las distribuciones de las preferencias de representantes y representados en dos dimensiones: autoposicionamiento ideológico en la escala izquierdaderecha y satisfacción con la democracia. Para el caso de Argentina, y como complemento al autoposicionamiento ideológico en la escala izquierda-derecha, también incluimos el autoposicionamiento en el eje peronismo-antiperonismo dada la configuración de su sistema de partidos. Probablemente, para un votante y representante argentino tenga más sentido autoposicionarse en este eje que en una escala izquierda-derecha (Lupu y Werner, 2017).

De acuerdo con este análisis descriptivo, Uruguay aparece como el país menos congruente considerando la escala ideológica. Los representantes no pasan del valor 7 en la escala ideológica, mientras que los ciudadanos se distribuyen normalmente, con una ligera polarización hacia ambos costados de la curva. Por otro lado, para el caso argentino, se observa mayor congruencia programática al emplear el eje peronista-antiperonista, en contraste con el eje izquierda-derecha. Esto se reproduce tanto en el análisis de medias como de distribuciones. El caso de Chile, en tanto, muestra la convivencia de una élite polarizada y de una ciudadanía moderada. Mientras la élite se carga hacia valores de izquierda, los ciudadanos siguen prefiriendo la opción 5 . El hecho de que la élite esté más polarizada obedece no sólo a que en 2013 la coalición de centro-izquierda superó ampliamente a la de derecha, sino que también es producto del sistema electoral binominal. Dado que se eligen dos escaños por distrito, las coaliciones aseguran un cupo con un tercio de los votos. Entonces, basta con que conquisten a ese tercio de izquierda o de derecha para acceder a un escaño, sin necesidad de avanzar hacia votantes moderados (Magar et al., 1998; Navia, 2005).

Esta primera evidencia descriptiva nos conduce a discutir la ecuación optimista que sostiene una relación lineal entre institucionalización y congruencia. Luna (2007) avanzó en este debate aunque desde una perspectiva exclusivamente teórica y bibliográfica. Sus planteamientos tienen sentido al someter la hipótesis rival al test estadístico. Esa hipótesis rival es justamente lo opuesto a la ecuación optimista. Dada la estabilidad de la competencia político-electoral en los sistemas institucionalizados, es plausible que las élites no teman perder adhesión al polarizarse en los ejes programáticos, pues los votantes cautivos las acompañarán a todo evento. En cambio, en sistemas más inestables y volátiles, es factible que los partidos emergentes busquen la congruencia con sus electores a fin de construir bases de apoyo mínimas para emprender una aventura electoral. A esto se suma un argumento institucional asociado al efecto de las reglas electorales. Como adelantamos, Uruguay es el país con el sistema más proporcional. Sin embargo, es el que exhibe los niveles más bajos de congruencia. Este hallazgo es consistente con la teoría (Blais y Bodet, 2006; Golder y Lloyd, 2014). Los sistemas más proporcionales generan condiciones propicias para una competencia electoral multidimensional. Por tanto, no sólo existen incentivos para la fragmentación partidaria, sino que también para la búsqueda de plataformas programáticas distinguibles del resto de los partidos en competencia. 
Figura 1

Congruencia programática en Argentina, Chile y Uruguay. Medida many-to-many

\begin{tabular}{|l|l|l|l|}
\hline \multicolumn{1}{|c|}{ Dimensión } & Argentina & Chile & \\
\hline $\begin{array}{l}\text { Eje izquierda- } \\
\text { derecha }\end{array}$ & & & \\
\hline $\begin{array}{l}\text { Eje peronismo- } \\
\text { antiperonismo }\end{array}$ & & & \\
\hline $\begin{array}{l}\text { Satisfacción con } \\
\text { la democracia }\end{array}$ & & & \\
\hline
\end{tabular}

Fuente: Elaboración propia con datos de IDRC (2013).

La Figura 2 compara los países utilizando la medición many-to-many. Como se señaló, esta medida compara las distribuciones de representantes y ciudadanos. Para ello, se emplea la función de distribución acumulada. Cuanto mayor sea la brecha entre representantes y representados, menor será la congruencia programática. Como se puede observar, Uruguay aparece como el país más incongruente en el eje ideológico. Siguiendo a Luna (2007), esta aparente anomalía -sobre todo por el carácter altamente institucionalizado del sistema de partidos uruguayo- obedece a representantes con mayor intensidad ideológica que los propios votantes, y en un eje de competencia entre el Frente Amplio y los partidos más tradicionales. Para Argentina, y como anunciamos, los resultados indican que la brecha entre representantes y votantes es sustancialmente mayor en el eje izquierdaderecha que en el eje peronismo-antiperonismo. Finalmente, Chile figura como el caso más congruente del Cono Sur al examinar el eje izquierda-derecha. Subrayamos que estos resultados preliminares toman las distribuciones generales de representantes y votantes sin discriminar por partido o coalición.

En cuanto a los niveles de satisfacción con la democracia, los tres casos se comportan de manera similar, aunque con una mayor brecha en el caso de Uruguay. La menor distancia entre las percepciones de representantes y representados sucede en Argentina, país con niveles de calidad de la democracia inferiores en comparación con Chile y Uruguay (Levine y Molina, 2007). Probablemente, lo que explique esta diferencia obedezca a las expectativas 
del régimen político en estos dos últimos casos. Es factible que los ciudadanos esperen más de la democracia y que al comparar esas expectativas con los resultados, la brecha con los representantes aumente. Dicho en otras palabras, mientras los representantes se encuentran satisfechos con el régimen que han ido construyendo, los ciudadanos sienten que la democracia no ha dado todos los frutos esperados en función de sus propias expectativas.

Figura 2

Congruencia programática en Argentina, Chile y Uruguay. Medida many-to-many

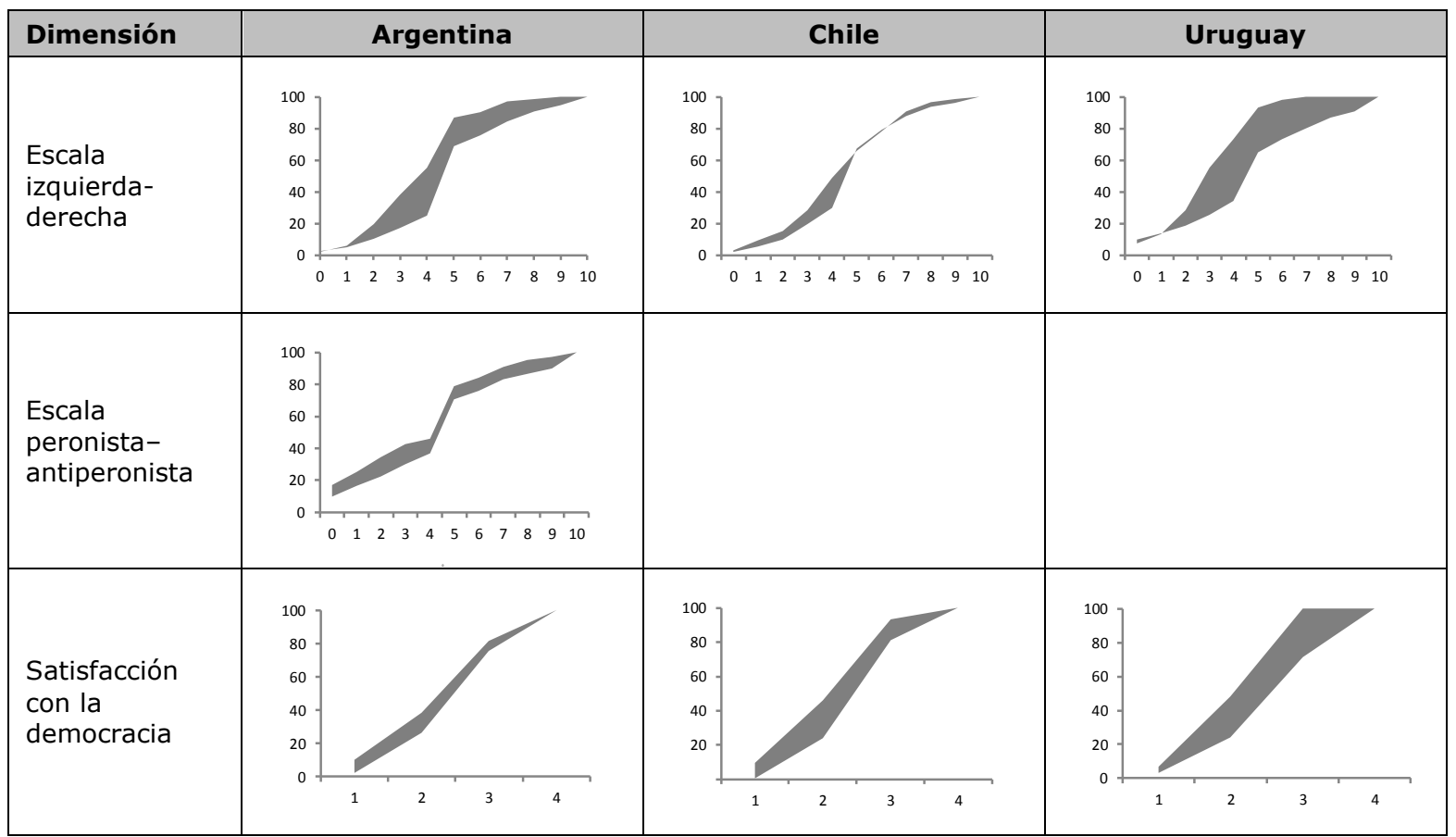

Fuente: Elaboración propia con datos de IDRC (2013).

¿Qué lecciones deja este análisis? Pareciera ser que una alta institucionalización del sistema de partidos puede convivir con bajos niveles de congruencia programática. El caso de Uruguay es prueba de aquello. El país aparece como el más incongruente en la mayoría de las mediciones y dimensiones utilizadas. Caso contrario ocurre con Chile, con un sistema de partidos también institucionalizado. Mientras tanto, Argentina figura con altos niveles de congruencia programática al menos en las dos dimensiones seleccionadas en el análisis, siendo un sistema de partidos menos institucionalizado que Uruguay y Chile.

Para explicar las variaciones de la congruencia programática, hemos construido modelos estadísticos lineales multivariados utilizando la medida many-to-one. La variable dependiente corresponde a la distancia absoluta entre un representante promedio y los ciudadanos. Construimos tres modelos por país. En el primero, tomamos un representante 
promedio del total de la élite. Luego, en los otros dos modelos lo hacemos con un representante promedio de los partidos o coaliciones más relevantes. Como variables independientes -guiadas por la teoría citada más arriba- incluimos algunas características sociodemográficas de los encuestados como sexo, edad, hábitat, nivel socioeconómico. Adicionalmente, elegimos algunas variables políticas como el interés en la política, la confianza en partidos y la identificación con algún partido o coalición. Nuestro propósito es conocer los determinantes sociodemográficos y políticos de la congruencia programática. La Tabla 3 muestra los datos descriptivos de cada variable por país (encuestas nacionales).

Tabla 3

Resumen de variables

\begin{tabular}{|c|c|c|c|c|c|c|c|c|c|}
\hline & \multicolumn{3}{|c|}{ Argentina } & \multicolumn{3}{|c|}{ Chile } & \multicolumn{3}{|c|}{ Uruguay } \\
\hline & $\mathbf{N}$ & Promedio & $\begin{array}{c}\begin{array}{c}\text { Desviación } \\
\text { estándar }\end{array} \\
\end{array}$ & $\mathbf{N}$ & Promedio & $\begin{array}{c}\begin{array}{c}\text { Desviación } \\
\text { estándar }\end{array} \\
\end{array}$ & $\mathbf{N}$ & Promedio & $\begin{array}{c}\text { Desviación } \\
\text { estándar }\end{array}$ \\
\hline $\begin{array}{l}\text { Interés en la } \\
\text { política }\end{array}$ & 1196 & 3,1 & 0,9 & 1156 & 3,3 & 0,85 & 1163 & 3,1 & 0,9 \\
\hline $\begin{array}{l}\text { Satisfacción con } \\
\text { la situación } \\
\text { económica }\end{array}$ & 1197 & 6,2 & 2,2 & 1188 & 6 & 2,22 & 1178 & 6,2 & 2,2 \\
\hline Sexo & 1200 & 1,5 & 0,5 & 1200 & 1,5 & 0,5 & 1202 & 1,5 & 0,5 \\
\hline Edad & 1200 & 44,6 & 17 & 1200 & 47,5 & 17,71 & 1202 & 45,2 & 17,8 \\
\hline $\begin{array}{l}\text { Nivel } \\
\text { Socioeconómico }\end{array}$ & 1200 & 3,6 & 0,9 & 1200 & 3,3 & 1,03 & 1202 & 3,8 & 1,7 \\
\hline Región & 1200 & 0,4 & 0,5 & 1200 & 0,5 & 0,5 & 1202 & 0,5 & 0,5 \\
\hline $\begin{array}{l}\text { Partido } \\
\text { Justicialista/ } \\
\text { Otros partidos }\end{array}$ & 1200 & 0,4 & 0,5 & & & & & & \\
\hline Alianza & & & & 1128 & 0,14 & 0,35 & & & \\
\hline Concertación & & & & 1200 & 0,23 & 0,42 & & & \\
\hline Partido Colorado & & & & & & & 1202 & 0,1 & 0,3 \\
\hline Frente Amplio & & & & & & & 1202 & 0,4 & 0,5 \\
\hline Partido Nacional & & & & & & & 1202 & 0,1 & 0,4 \\
\hline
\end{tabular}

Fuente: Elaboración propia con datos de IDRC (2013).

Para interpretar los modelos es necesario considerar lo siguiente. La variable dependiente -como señalamos- es la distancia entre el representante promedio y un encuestado. Mientras mayor sea la distancia, habrá menos congruencia. Por tanto, si el coeficiente de alguna de las variables es positivo y significativo, indicará que un incremento en los valores de esa variable implica mayor incongruencia. La Tabla 4 muestra los resultados para Argentina. Los valores de $\mathrm{R}$ cuadrado son bajos, pero permiten realizar algunas interpretaciones. En el modelo 1 -que considera un representante promedio- una de las variables significativa es el lugar de residencia del encuestado. Los códigos de esa variable corresponden a 0 para personas que no viven en el Gran Buenos Aires, y 1 para las personas que viven en el Gran Buenos Aires. Dado que el coeficiente es negativo, entonces el modelo indica que hay más congruencia entre el representante promedio y los encuestados del Gran 
Buenos Aires en comparación con los encuestados que no habitan en el Gran Buenos Aires. Por edad, en tanto, un incremento de esta variable implica mayor incongruencia. Como era de esperar, los votantes identificados con el PJ tienen mayor congruencia con los representantes de su partido. El modelo 2 indica resultados que van en la misma dirección. Es decir, que existe mayor congruencia entre los representantes del PJ y los habitantes del Gran Buenos Aires en comparación con quienes habitan en otras provincias. De igual forma, incrementos en la edad van asociados a mayores dosis de incongruencia programática. En el modelo 3, que considera a representantes de distintos partidos, la edad deja de ser un predictor robusto, sobreviviendo solamente el hábitat de los encuestados que mantiene idéntica tendencia en comparación con los dos modelos. Es decir, que existe mayor congruencia entre representantes de otros partidos y los habitantes del Gran Buenos Aires.

De acuerdo con estos datos, entonces, si bien para Argentina se advierten altos niveles de congruencia programática entre representantes y votantes, dicha congruencia está claramente segmentada por el lugar de residencia de los encuestados. A pesar de que Argentina es un estado federal, los mayores niveles de congruencia se dan en el Gran Buenos Aires. Sorprende, eso sí, que en los tres modelos esta variable asuma valores similares y en la misma dirección, haciendo ver que en el resto de Argentina es mayor la desconexión entre representantes y votantes.

Esta interpretación cambia cuando se utiliza el eje peronismo-antiperonismo. En primer lugar, desaparece el efecto del hábitat. En segundo lugar, sobrevive el efecto de la edad con un comportamiento muy similar a lo observado en el eje izquierda-derecha. En tercer lugar, emergen algunas variables asociadas a percepciones políticas y económicas. Por ejemplo, la identificación de los ciudadanos con el PJ incrementa los niveles de congruencia tanto en el modelo 1 (representante promedio) como en el modelo 2 (representantes del PJ). El efecto de esta variable es sustantivamente mayor en comparación con el modelo que toma como variable dependiente al eje izquierda-derecha. Luego, aparecen como predictores el interés en la política y la satisfacción con la situación económica. En el caso del interés en la política, un mayor interés favorece la congruencia programática en el modelo 2 donde se considera un representante promedio del PJ. Este efecto se invierte en el modelo 3 correspondiente a representantes de otros partidos. Por otro lado, cuanto mayor satisfacción económica, mayor congruencia tanto en el modelo general como en el modelo aplicado sólo a representantes del PJ. Adicionalmente, observamos el efecto del nivel socioeconómico de las personas. Luego de probar que satisfacción económica y nivel socioeconómico no produjeron problemas de multicolinealidad en el modelo, los resultados indican que, al menos en el modelo 3, la congruencia entre representantes de otros partidos y los ciudadanos se da con mayor fuerza en los segmentos más altos. 


\section{Tabla 4 \\ Argentina, modelo de regresión lineal múltiple: escala ideológica izquierda-derecha. Variable dependiente: Congruencia programática}

\begin{tabular}{|c|c|c|c|c|c|c|}
\hline \multirow{3}{*}{ Variables } & \multicolumn{3}{|c|}{ Eje izquierda-derecha } & \multicolumn{3}{|c|}{ Eje peronismo-antiperonismo } \\
\hline & Modelo 1 & Modelo 2 & Modelo 3 & Modelo 1 & Modelo 2 & Modelo 3 \\
\hline & $\begin{array}{l}\text { Modelo } \\
\text { General }\end{array}$ & $\begin{array}{c}\text { Representantes } \\
\text { PJ }\end{array}$ & $\begin{array}{c}\text { Representantes } \\
\text { otros partidos }\end{array}$ & $\begin{array}{l}\text { Modelo } \\
\text { General }\end{array}$ & \begin{tabular}{|c|} 
Representantes \\
PJ
\end{tabular} & $\begin{array}{c}\text { Representantes } \\
\text { otros partidos }\end{array}$ \\
\hline $\begin{array}{l}\text { Sexo ( } 0=\text { Hombres; } \\
1=\text { Mujer })\end{array}$ & $\begin{array}{l}-0.0279 \\
(0.103) \\
\end{array}$ & $\begin{array}{l}-0.0320 \\
(0.103) \\
\end{array}$ & $\begin{array}{l}-0.0192 \\
(0.102) \\
\end{array}$ & $\begin{array}{c}0.149 \\
(0.114) \\
\end{array}$ & $\begin{array}{c}0.131 \\
(0.136) \\
\end{array}$ & $\begin{array}{c}0.187 \\
(0.121) \\
\end{array}$ \\
\hline Edad & $\begin{array}{l}0.00603 * \\
(0.00312)\end{array}$ & $\begin{array}{l}0.00757 * * \\
(0.00314) \\
\end{array}$ & $\begin{array}{c}0.00481 \\
(0.00309) \\
\end{array}$ & \begin{tabular}{|c|}
$0.0103 * * *$ \\
$(0.00339)$ \\
\end{tabular} & $\begin{array}{l}-0.00436 \\
(0.00405) \\
\end{array}$ & $\begin{array}{l}0.0176 * * * \\
(0.00361)\end{array}$ \\
\hline $\begin{array}{l}\text { Región }(1=\mathrm{GBA} ; \\
0=\text { otros })\end{array}$ & $\begin{array}{c}-0.239 * * \\
(0.104) \\
\end{array}$ & $\begin{array}{c}-0.292 * * * \\
(0.105) \\
\end{array}$ & $\begin{array}{c}-0.208 * * \\
(0.103)\end{array}$ & $\begin{array}{l}-0.0341 \\
(0.116) \\
\end{array}$ & $\begin{array}{l}0.0509 \\
(0.138) \\
\end{array}$ & $\begin{array}{l}-0.153 \\
(0.123) \\
\end{array}$ \\
\hline $\begin{array}{l}\text { Nivel socioeconómico } \\
(1=E ; 7=A B)\end{array}$ & $\begin{array}{l}-0.0519 \\
(0.0574)\end{array}$ & $\begin{array}{l}-0.0504 \\
(0.0578)\end{array}$ & $\begin{array}{l}-0.0471 \\
(0.0568)\end{array}$ & $\begin{array}{c}0.0499 \\
(0.0641)\end{array}$ & $\begin{array}{l}0.205^{* * *} \\
(0.0766)\end{array}$ & $\begin{array}{l}-0.0545 \\
(0.0682)\end{array}$ \\
\hline $\begin{array}{l}\text { Interés en la política } \\
\text { (1=Muy interesado; } \\
\text { 4=Nada interesado) }\end{array}$ & $\begin{array}{l}-0.110 * \\
(0.0566) \\
\end{array}$ & $\begin{array}{l}-0.0436 \\
(0.0570) \\
\end{array}$ & $\begin{array}{c}-0.154 * * * \\
(0.0560) \\
\end{array}$ & $\begin{array}{l}-0.113^{*} \\
(0.0636) \\
\end{array}$ & $\begin{array}{l}0.154 * * \\
(0.0761) \\
\end{array}$ & $\begin{array}{c}-0.294 * * * \\
(0.0678) \\
\end{array}$ \\
\hline $\begin{array}{l}\text { Percepción situación } \\
\text { económica del país } \\
\text { (1=Muy bueno/ } \\
5=\text { Muy mala) }\end{array}$ & $\begin{array}{l}0.0145 \\
(0.0247)\end{array}$ & $\begin{array}{c}0.0163 \\
(0.0248)\end{array}$ & $\begin{array}{c}0.0106 \\
(0.0244)\end{array}$ & $\begin{array}{l}0.00920 \\
(0.0273)\end{array}$ & $\begin{array}{c}0.0525 \\
(0.0326)\end{array}$ & $\begin{array}{l}-0.0269 \\
(0.0290)\end{array}$ \\
\hline $\begin{array}{l}\text { Partido Justicialista } \\
(1=\mathrm{PJ} ; 0=\text { resto })\end{array}$ & $\begin{array}{l}0.0377 \\
(0.106) \\
\end{array}$ & $\begin{array}{l}-0.0378 \\
(0.107) \\
\end{array}$ & $\begin{array}{l}0.0971 \\
(0.105) \\
\end{array}$ & \begin{tabular}{|c|}
$-0.358 * * *$ \\
$(0.117)$ \\
\end{tabular} & $\begin{array}{c}-1.821 * * * \\
(0.140) \\
\end{array}$ & $\begin{array}{c}0.679 * * * \\
(0.125) \\
\end{array}$ \\
\hline Constante & $\begin{array}{c}2.105^{* * *} \\
(0.402)\end{array}$ & $\begin{array}{c}2.125^{* * *} \\
(0.405)\end{array}$ & $\begin{array}{c}2.138 * * * \\
(0.398)\end{array}$ & $\begin{array}{c}1.914 * * * \\
(0.455)\end{array}$ & $\begin{array}{c}2.658 * * * \\
(0.544)\end{array}$ & $\begin{array}{c}2.099 * * * \\
(0.485)\end{array}$ \\
\hline Observaciones & 851 & 851 & 851 & 910 & 910 & 910 \\
\hline $\mathrm{R}^{2}$ & 0.015 & 0.017 & 0.018 & 0.024 & 0.198 & 0.089 \\
\hline
\end{tabular}

Fuente: Elaboración propia con datos de la encuesta IDRC (2013).

Errores estándar entre paréntesis.

$* * * \mathrm{p}<0.01, * * \mathrm{p}<0.05, * \mathrm{p}<0.1$.

En el caso de Chile los modelos, al igual que en Argentina, se especifican en tres etapas (ver Tabla 5). Primero, un modelo general con un representante promedio. Luego, con un representante de la coalición de centro-derecha denominada Alianza. El tercer modelo toma a un representante promedio de la coalición de centro-izquierda, Nueva Mayoría. La variable que mide la identificación con coaliciones fue recodificada en tres valores: Nueva Mayoría, Alianza y no identificados. En los modelos es esta última categoría la que opera como de referencia

La capacidad explicativa de estos modelos es sustancialmente superior respecto a Argentina. Los predictores más robustos son la edad y la identificación con alguna de las dos grandes coaliciones. En el caso de la edad, y al igual que en Argentina, incrementos en la variable van asociados a menor congruencia programática. En el caso de la identificación con coaliciones, en tanto, los resultados son los esperados, aunque con algunas especificidades. En el modelo 1 la identificación de los encuestados con alguna de las dos coaliciones no es un predictor de congruencia programática. En el modelo 2, que toma un representante 
promedio de la Alianza, la congruencia es más fuerte con sus electores. Mientras tanto, en el modelo 3 -representantes de la NM- aparece como predictor la identificación con la Alianza, pero no con los votantes de la NM.

\section{Tabla 5
Chile, modelo de regresión lineal múltiple: ideología Variable dependiente: congruencia programática}

\begin{tabular}{|l|c|c|c|}
\hline \multirow{2}{*}{ Variables } & $\mathbf{( 1 )}$ & $\mathbf{( 2 )}$ & $\mathbf{( 3 )}$ \\
\cline { 2 - 4 } & Modelo General & $\begin{array}{c}\text { Modelo } \\
\text { Alianza }\end{array}$ & $\begin{array}{c}\text { Modelo Nueva } \\
\text { Mayoría }\end{array}$ \\
\hline Interés en la política (1=Muy interesado; 4=Nada & -0.0493 & $-0.171^{* * *}$ & 0.0419 \\
interesado) & $(0.0611)$ & $(0.0612)$ & $(0.0612)$ \\
\hline Percepción situación económica del país (1=Muy & -0.0173 & -0.0257 & -0.00857 \\
bueno/5=Muy mala) & $(0.0246)$ & $(0.0247)$ & $(0.0247)$ \\
\hline Sexo (1=hombre; 2=mujer) & -0.104 & -0.149 & -0.0391 \\
& $(0.106)$ & $(0.106)$ & $(0.106)$ \\
\hline Edad & 0.00393 & -0.000468 & 0.00467 \\
& $(0.00309)$ & $(0.00310)$ & $(0.00310)$ \\
\hline Nivel socioeconómico (1=Alto; 4=Bajo) & -0.0147 & 0.0558 & -0.0365 \\
& $(0.0555)$ & $(0.0556)$ & $(0.0557)$ \\
\hline Alianza (1=alianza; 0=otros) & $1.129 * * *$ & $-0.330 * *$ & $1.456 * * *$ \\
& $(0.142)$ & $(0.142)$ & $(0.142)$ \\
\hline Nueva Mayoría (1=NM; 0=resto) & 0.0934 & $0.785 * * *$ & $-0.462 * * *$ \\
& $(0.121)$ & $(0.121)$ & $(0.121)$ \\
\hline Constante & $1.200 * * *$ & $2.180^{* * *}$ & $1.403^{* * *}$ \\
\hline Observaciones & $(0.374)$ & $(0.375)$ & $(0.375)$ \\
\hline $\mathrm{R}^{2}$ & 685 & 685 & 685 \\
\hline
\end{tabular}

Fuente: Elaboración propia con datos de la encuesta IDRC (2013).

Errores estándar entre paréntesis.

$* * * \mathrm{p}<0.01, * * \mathrm{p}<0.05, * \mathrm{p}<0.1$.

En el caso de Uruguay hay tres resultados relevantes. El primero es que la pertenencia a algún partido político es un predictor robusto de la congruencia programática. Dado que la identificación con partidos es más fuerte hacia el Frente Amplio, entonces el modelo arroja un coeficiente negativo y significativo, señalando que la distancia entre representantes y representados disminuye cuando el encuestado se identifica con el Frente Amplio (ver Tabla 6). A diferencia de Argentina y Chile, los niveles de congruencia sistemáticamente responden al nivel socioeconómico de los encuestados, siendo mayor en los estratos de más ingresos. Esto último aplica tanto al modelo general, como a los modelos para el Partido Colorado, Partido Nacional y Frente Amplio. 


\section{Tabla 6 \\ Uruguay, modelo de regresión lineal múltiple: ideología Variable dependiente: congruencia programática}

\begin{tabular}{|c|c|c|c|c|}
\hline & (1) & (2) & (3) & (4) \\
\hline Variables & $\begin{array}{l}\text { Modelo } \\
\text { General }\end{array}$ & $\begin{array}{c}\text { Congresistas } \\
\text { Partido } \\
\text { Colorado }\end{array}$ & $\begin{array}{c}\text { Congresistas } \\
\text { Partido } \\
\text { Nacional }\end{array}$ & $\begin{array}{c}\text { Congresistas } \\
\text { Frente } \\
\text { Amplio }\end{array}$ \\
\hline $\begin{array}{l}\text { Interés en la política } \\
\text { ( } 1=\text { Muy interesado; } 4=\text { Nada interesado) }\end{array}$ & $\begin{array}{l}-0.0293 \\
(0.0575) \\
\end{array}$ & $\begin{array}{l}-0.0911 \\
(0.0612)\end{array}$ & $\begin{array}{l}-0.112^{*} \\
(0.0644)\end{array}$ & $\begin{array}{l}-0.00400 \\
(0.0597)\end{array}$ \\
\hline $\begin{array}{l}\text { Percepción situación económica del país } \\
\text { ( } 1=\text { Muy bueno/5=Muy mala) }\end{array}$ & $\begin{array}{l}0.0569 * * \\
(0.0234)\end{array}$ & $\begin{array}{c}0.0321 \\
(0.0249) \\
\end{array}$ & $\begin{array}{c}0.0234 \\
(0.0262) \\
\end{array}$ & $\begin{array}{c}0.0722 * * * \\
(0.0243) \\
\end{array}$ \\
\hline Sexo (1=hombre; 2 =mujer) & $\begin{array}{c}0.107 \\
(0.0991) \\
\end{array}$ & $\begin{array}{l}0.0531 \\
(0.105) \\
\end{array}$ & $\begin{array}{c}0.0382 \\
(0.111) \\
\end{array}$ & $\begin{array}{c}0.138 \\
(0.103) \\
\end{array}$ \\
\hline Edad & $\begin{array}{c}0.000656 \\
(0.00288) \\
\end{array}$ & $\begin{array}{c}0.00146 \\
(0.00307)\end{array}$ & $\begin{array}{c}0.00133 \\
(0.00323)\end{array}$ & $\begin{array}{c}-0.000456 \\
(0.00300) \\
\end{array}$ \\
\hline $\begin{array}{l}\text { Nivel socioeconómico } \\
\text { (1=Muy bajo; 7=Muy alto) }\end{array}$ & $\begin{array}{c}-0.186 * * * \\
(0.0315)\end{array}$ & $\begin{array}{c}-0.186 * * * \\
(0.0335) \\
\end{array}$ & $\begin{array}{c}-0.175 * * * \\
(0.0352) \\
\end{array}$ & $\begin{array}{c}-0.172 * * * \\
(0.0327) \\
\end{array}$ \\
\hline Región ( $1=$ Montevideo; $0=$ resto) & $\begin{array}{l}-0.0961 \\
(0.102)\end{array}$ & $\begin{array}{c}-0.117 \\
(0.109)\end{array}$ & $\begin{array}{c}-0.123 \\
(0.114)\end{array}$ & $\begin{array}{l}-0.0895 \\
(0.106)\end{array}$ \\
\hline Partido Colorado ( $1=\mathrm{PC} ; 0=$ otros $)$ & $\begin{array}{c}1.855^{* * * *} \\
(0.169)\end{array}$ & $\begin{array}{c}1.597 * * * \\
(0.180)\end{array}$ & $\begin{array}{c}1.426 * * * \\
(0.189)\end{array}$ & $\begin{array}{c}1.994 * * * \\
(0.176)\end{array}$ \\
\hline Frente Amplio ( $1=\mathrm{FA} ; 0=$ otros) & $\begin{array}{c}-0.502 * * * \\
(0.122) \\
\end{array}$ & $\begin{array}{c}0.574 * * * \\
(0.130) \\
\end{array}$ & $\begin{array}{c}1.007 * * * \\
(0.137) \\
\end{array}$ & $\begin{array}{c}-0.989 * * * \\
(0.127) \\
\end{array}$ \\
\hline Partido Nacional ( $1=\mathrm{PN} ; 0=$ otros) & $\begin{array}{c}1.652 * * * \\
(0.159)\end{array}$ & $\begin{array}{c}1.350 * * * \\
(0.169)\end{array}$ & $\begin{array}{c}1.160 * * * \\
(0.178)\end{array}$ & $\begin{array}{c}1.796 * * * \\
(0.165)\end{array}$ \\
\hline Constante & $\begin{array}{c}2.763 * * * \\
(0.350) \\
\end{array}$ & $\begin{array}{c}2.217^{* * *} \\
(0.372) \\
\end{array}$ & $\begin{array}{c}2.061^{* * *} \\
(0.392) \\
\end{array}$ & $\begin{array}{c}.195^{* * *} \\
(0.363)\end{array}$ \\
\hline Observaciones & 999 & 999 & 999 & 999 \\
\hline $\mathrm{R}^{2}$ & 0.296 & 0.141 & 0.117 & 0.368 \\
\hline
\end{tabular}

Fuente: Elaboración propia con datos de la encuesta IDRC (2013).

Errores estándar entre paréntesis.

$* * * \mathrm{p}<0.01, * * \mathrm{p}<0.05, * \mathrm{p}<0.1$.

\section{Conclusiones}

Los resultados indican que los sistemas de partidos clasificados por la literatura como altos en institucionalización pueden convivir con niveles de congruencia programática dispares. La forma en que se vinculan representantes y ciudadanos parece ser independiente de la institucionalización de los sistemas de partidos.

Es difícil empalmar teóricamente estos hallazgos con la literatura más tradicional sobre institucionalización de los sistemas de partidos. Dado que uno de los indicadores que generalmente se utiliza para medir institucionalización es la volatilidad electoral, resulta curioso que en países más estables existan menores niveles de congruencia programática entre élite y ciudadanía. Dicho en otras palabras, parece ser que en sistemas estables las preferencias electorales se mantienen a pesar de la distancia entre élite y votantes en algunos ejes de competencia.

Sin embargo, la teoría referenciada más arriba ayuda entender este resultado. Desde una perspectiva institucional, los sistemas más proporcionales generan incentivos para la fragmentación y para la búsqueda de espacios programáticos por parte de líderes y partidos. 
Por eso mismo, se produce una distancia natural entre el votante mediano y las posturas que esos partidos defienden. En el afán por distinguirse de otros dada la oferta electoral, los partidos buscan posiciones más extremas. Visto así, entonces, no sorprende tanto la convivencia de sistemas de partidos institucionalizados con altos niveles de incongruencia programática. Precisamente porque las preferencias de los electores están ancladas en un ambiente de alta raigambre social de los partidos, las élites se sienten con la suficiente libertad para polarizar los debates programáticos, confirmando así su postura frente a electores ideológicamente intensos.

En definitiva, las variaciones en el volumen de la congruencia programática, cualquiera sea la medición, no dicen mucho -por sí solas- acerca de la estabilidad de un sistema de partidos y, menos, de sus niveles de institucionalización. Claro está que la convivencia entre alta incongruencia y bajos niveles de institucionalización es dañina para la estabilidad democrática, pues supone una sistemática rotación de partidos en conjunto con votantes distanciados ideológicamente. Algo similar ocurre en sistemas escasamente institucionalizados, pero con altos niveles de congruencia programática. Es posible que esa congruencia responda a la necesidad de los partidos emergentes para representar los intereses de los votantes, pero como esos partidos son de corta duración, entonces la congruencia programática se torna muy circunstancial.

Los principales resultados de los modelos estadísticos diseñados para explicar las variaciones de congruencia indican fuertes diferencias entre los casos. En Argentina, tomando como variable dependiente la congruencia en el eje izquierda-derecha, existe un fuerte componente territorial. La congruencia es más fuerte en Buenos Aires en comparación con el resto del país. En Chile y Uruguay, en tanto, una de las variables más robustas para explicar las variaciones de congruencia corresponde a la identificación partidaria o la identificación con alguna de las coaliciones, cuestión que probablemente se deba a los mayores niveles de institucionalización del sistema de partidos en comparación con Argentina. Lo que hace distintivo a Uruguay de los otros casos corresponde al efecto del nivel socioeconómico, existiendo mayor congruencia en los estratos altos en comparación con los estratos bajos.

En síntesis, el artículo da ciertas pistas sobre el análisis de la congruencia programática desde su volumen y composición. A nivel descriptivo, la estrategia many-tomany permitió comparar los casos en función de distribuciones de preferencias, medida más útil que la mera comparación de promedios. Adicionalmente, y con el fin de analizar la composición de la congruencia, utilizamos la estrategia many-to-one. El objetivo acá fue identificar los determinantes de la congruencia programática a partir de las características socioeconómicas y políticas de los ciudadanos. Entonces, si la estrategia many-to-many ayuda a comparar los volúmenes o magnitudes de la congruencia programática, la estrategia many-to-one contribuye a caracterizar su composición. 


\section{Referencias bibliográficas}

ACHEN, C. "Measuring representation: perils of the correlation coefficient". American Journal of Political Science, vol. 21, n 4, 1977.

"Measuring representation". American Journal of Political Science, vol. 22, n 3, 1978.

ADAMS, J. Party competition and responsible party government. Ann Arbor: University of Michigan Press, 2001.

AMES, B.; SMITH, E. "Knowing left from right: ideological identification in Brazil, 2002-2006". Journal of Politics in Latin America, vol. 3, no 2, 2010.

BELCHIOR, A. "Ideological congruence among European political parties". The Journal of Legislative Studies, vol. 16, $\mathrm{n}^{\circ} 1,2010$.

BLAIS, A.; BODET, M. "Does proportional representation foster closer congruence between citizens and policymakers?". Comparative Political Studies, vol. 36, 2006.

BuQuet, D.; Selios, L. Political congruence in Uruguay. In: Fuentes, C.; JoIgnant, A.; Morales, M. (eds.). Malaise in representation in Latin American countries: Chile, Uruguay and Argentina.

Basingstoke: Palgrave, 2017.

DALTON, J. "Political parties and political representation: party supporters and party elites in nine nations". Comparative Political Studies, vol. 18, n 3, 1985.

España-NÁJera, A.; MarTínez, M. "Political representation in Central America". Latin Americas Studies Association Congress, 2010.

Giger, N.; AinA, G.; LefKOfRIDI, Z. "Ideological congruence and voter tournout". Conferencia Tag der Politikwissenschaft, 2011.

GOLDER, M.; LLOYD, G. "Re-evaluating the relationship between electoral rules and ideological congruence". European Journal of Political Research, vol. 53, no 1, 2014.

Golder, M.; StRAMSKI, J. "Ideological congruence and electoral institutions". American Journal of Political Science, vol. 54, n 1, 2010.

HAWKINS, K.; MORGENSTERN, S. "Cohesion of legislators in Latin America: patterns and explanations". Latin Americas Studies Association Congress, 2000.

Huber, J.; POWELL, B. "Congruence between citizens and policymakers in two visions of liberal democracy". World Politics, vol. 46, no 3, 1994.

KITSCHELT, H. "Linkages between citizens and politicians in democratic polities". Comparative Political Studies, vol. 33, $\mathrm{n}^{\circ} 6,2000$.

KITSCHELT, H., et al. Post-communist party systems: competition, representation, and inter-party cooperation. New York: Cambridge University Press, 1999.

Latin American party systems. New York: Cambridge University Press, 2010.

LeVInE, D.; Molina, J. "La calidad de la democracia en América Latina: una visión comparada". América Latina Hoy, vol. 45, 2007.

. The quality of democracy in Latin America. Boulder: Lynne Rienner Publishers, 2011. 
CONGRUENCIA PROGRAMÁTICA EN EL CONO SUR. ARGENTINA, CHILE Y URUGUAY EN PERSPECTIVA COMPARADA

LUNA, J. "Representación política en América Latina: el estado de la cuestión y una propuesta de agenda". Política y Gobierno, vol. 14, n², 2007.

LUNA, J.; ZECHMEISTER, E. "The quality of representation in Latin America: a study of elite-mass congruence in nine countries". Comparative Political Studies, vol. 38, n 4, 2005.

. "Political representation in Latin America". In: KITSCHELT, H., et al. (eds.). Latin American party systems. Cambridge: Cambridge University Press, 2010.

LUPU, N.; WERNER, Z. "Mass-elite congruence and representation in Argentina". In: FUENTES, C.; Joignant, A.; Morales, M. (eds.). Malaise in representation in Latin American Countries: Chile, Uruguay and Argentina. Basingstoke: Palgrave, 2017.

Magar, E.; Rosenblum, M.; SAmuels, D. "On the absence of centripetal incentives in double-member districts. The case of Chile". Comparative Political Studies, vol. 31, n 6, 1998.

MAINWARING, S.; SCULlY, T. "La institucionalización de los sistemas de partidos en América Latina". Revista de Ciencia Política, vol. 17, n 1, 1995.

MAINWARING, S.; TORCAL, M. "La institucionalización de los sistemas de partidos y la teoría del sistema partidista después de la tercera ola democratizadora". América Latina Hoy, vol. 41, 2005.

Miller, W.; StOKeS, D. "Constituency influence in Congress". American Political Science Review, vol. $57, n^{\circ} 1,1963$.

MORALES, M. "Congruencia programática entre partidos y votantes en Chile". Perfiles Latinoamericanos, vol. 22, no 44, 2014.

MoRGAN, D. "Paradigms lost and pragmatism regained". Journal of Mixed Methods Research, vol. 1, n $1,2007$.

NAviA, P. "La transformación de votos en escaños: leyes electorales en Chile, 1833-2004". Política y Gobierno, vol. 12, n², 2005.

O’DonNeLL, G. "Delegative democracy". Journal of Democracy, vol. 5, n 1, 1994.

PAYNE, M., et al. ¿La política importa? Democracia y desarrollo en América Latina. Washington: Banco Interamericano de Desarrollo, 2003.

PITKIN, H. The concept of representation. Berkeley: University of California Press, 1967.

POWELL, B. "Experimental analysis of the social value of flocking by starlings (sturnus vulgaris) in relation to predation and foraging". Animal Behaviour, vol. 22, no 2, 1974.

. Elections as instruments of democracy: majoritarian and proportional visions. New Haven and London: Yale University Press, 2001. no $1,2004$.

"Political representation in comparative politics". Annual Review of Political Science, vol. 7,

"The ideological congruence controversy: the impact of alternatives measures, data, and time series periods on the effects of election rules". Comparative Political Studies, vol. $42, n^{\circ} 12$, 2009.

PrzeWorski, A.; ORTegA, J.; Gordon, S. "Una defensa de la concepción minimalista de la democracia". Revista Mexicana de Sociología, vol. 59, n 3, 1997. 
PrzeWorski, A.; STOKes, S.; BeRnARD, M. Democracy, accountability and representation. New York: Cambridge University Press, 1999.

SChmitt, H.; ThOMASSEN, J. (eds.). Political representation and legitimacy in the European Union. Oxford: Oxford University Press, 1999.

SiAvelis, P. "Enclaves de la transición y la democracia chilena". Revista de Ciencia Política, vol. 29, nº $1,2009$.

STOKES, S. Mandates and democracy: neoliberalism by surprise in Latin America. Cambridge University Press: New York, 2001.

WARWICK, P. "The ideological congruence illusion: the impact of violence". Legislative Studies Quarterly, vol. 41, n 1, 2016.

WESSELS, B. "System characteristics matter: empirical evidence from ten representation studies". In: Miller, W.; Pierce, R.; ThOMASSen, J. (eds.). Policy representation in Western democracies. Oxford: Oxford University Press, 1999.

WeYLAND, K. "Neoliberalism and democracy in Latin America: a mixed record". Latin American Politics and Society, vol. 46, $\mathrm{n}^{\circ} 1,2004$. 


\section{Resumo}

Congruência programática no Cone Sul. Argentina, Chile e Uruguai em perspectiva comparada

Os sistemas de partidos institucionalizados podem coexistir com uma alta incongruência programática entre a elite e os cidadãos. Sistemas partidários menos institucionalizados, pelo contrário, poderiam estar em posição de gerar maior congruência programática. Analisamos essa aparente anomalia no Cone Sul da América Latina, comparando Argentina, Chile e Uruguai. Metodologicamente, usamos três medidas de congruência: médias, comparação de distribuições (many-to-many), distância entre eleitores e um representante típico (many-toone). Nesta última medida, projetamos vários modelos de regressão para identificar as variáveis que predizem maior congruência. Concluímos que em sistemas estáveis as preferências eleitorais permanecem apesar da distância entre a elite e os eleitores em algumas áreas de competição, sendo o Uruguai o caso mais representativo.

Palavras-chave: congruência programática; sistemas partidários; institucionalização; Cone Sul; elite

\section{Abstract}

Programmatic congruence in the Southern Cone. Argentina, Chile, and Uruguay in comparative perspective

Institutionalized party systems can coexist with a low programmatic congruence between elites and citizens. To the contrary, less institutionalized party systems could be in a position to generate greater programmatic congruence. We analyze this apparent anomaly in the Southern Cone of Latin America, comparing Argentina, Chile, and Uruguay. Methodologically, we used three measures of congruence: averages, comparison of distributions (many-to-many), distance between voters and a median legislator (many-to-one). For this last measure, we designed multiple regression models to identify the variables that predict greater congruence. We arrived at the conclusion that in stable systems the electoral preferences remain despite the distance between elites and voters in some areas of the competition-Uruguay being the most representative case.

Keywords: programmatic congruence; party systems; institutionalization; Southern Cone; elite

\section{Résumé}

La congruence programmatique dans le Cône Sud. L'Argentine, le Chili et l'Uruguay dans une perspective comparative

Les systèmes de partis institutionnalisés peuvent coexister avec une forte incongruité programmatique entre I'élite et les citoyens. Au contraire, les systèmes partisans moins institutionnalisés pourraient être capables de générer une plus grande congruence programmatique. Nous analysons cette anomalie apparente dans le Cône Sud de I'Amérique Latine, en comparant I'Argentine, le Chili et I'Uruguay. Méthodologiquement, nous utilisons trois mesures de congruence: les moyennes, la comparaison des distributions (many-to-many), la distance entre les électeurs et un représentant typique (many-to-one). Sur cette dernière mesure, nous concevons des modèles de régression multiple pour identifier les variables qui prédisent une plus grande congruence. Nous concluons que dans les systèmes stables, les préférences électorales demeurent malgré la distance entre l'élite et les électeurs dans certaines zones de compétition, I'Uruguay étant le cas le plus représentatif.

Mots-clés: congruence programmatique; systèmes de partis; institutionnalisation; Cône Sud; élite

Artigo submetido à publicação em 19 julho de 2017. Versão final aprovada em 16 abril de 2018.

Opinião Pública adota a licença Creative Commons CC-BY.



